\title{
Primary intraosseous carcinoma of mandible: An update on review of literature with a case report
}

\author{
Shambhulingappa $\mathbf{P}^{1}$, Sheikh S ${ }^{2}$, Puri $^{3}{ }^{3}$, Jindal SK ${ }^{4}$ \\ ${ }^{1}$ B.D.S., M.D.S., Professor, Department of Oral Medicine and Radiology, M.M. College of Dental Sciences \& Research, Mulla- \\ na. \\ ${ }^{2}$ B.D.S., M.D.S., Professor, Department of Oral Medicine and Radiology, M.M. College of Dental Sciences \& Research, Mulla- \\ na. \\ ${ }^{3}$ B.D.S., P.G. Student, Department of Oral Medicine and Radiology, M.M. College of Dental Sciences \& Research, Mullana. \\ ${ }^{4}$ B.D.S., M.D.S., Senior Lecturer, Department of Oral Medicine and Radiology, Bhojia Dental College \& Hospital, Baddi, Hima- \\ chal Pradesh.
}

Correspondence:

Dr. Shambhulingappa P.,

Department of Oral Medicine and Radiology,

M. M. College of Dental Sciences and Research,

Mullana, District Ambala, Haryana-133203, India

E-mail: jindal2003@yahoo.com

\author{
Shambhulingappa P, Sheikh S, Puri N, Jindal SK. Primary intraosseous \\ carcinoma of mandible: An update on review of literature with a case \\ report. J Clin Exp Dent. 2010;2(2):e91-5. \\ http://www.medicinaoral.com/odo/volumenes/v2i2/jcedv2i2p91.pdf \\ Article Number: $678942 \quad$ http://www.medicinaoral.com/odo/indice.htm \\ C Medicina Oral S. L. C.I.F. B 96689336 - eISSN: 1989-5488 \\ eMail: jced@jced.es
}

\begin{abstract}
Primary intraosseous carcinoma (PIOC) is a rare malignant neoplasm of the jaws which is locally aggressive with quite poor prognosis. Overall and disease free survival is poor with almost $50 \%$ patients failing within first 2 years of follow up and reason can be attributed to the delayed diagnosis. But knowledge of the clinical, radiographic and histopathologic features of PIOC allows accurate and early diagnosis of the lesion so that an early and appropriate treatment can be instituted for better prognosis. Here a typical case of PIOC of the mandible is presented and an attempt is made to discuss all possible differential diagnosis and an update on review of literature is presented. Our case highlights that radiographic examination is one of the most effective methods for detecting early lesions of PIOCs.
\end{abstract}

Key words: Primary intraosseous carcinoma, mandible, panoramic radiography. 


\section{Introduction}

Primary intraosseous carcinoma (PIOC) was first described by Loos in 1913 as a central epidermoid carcinoma of the jaw. Willis in 1948 renamed it as an intraalveolar epidermoid carcinoma. It was Pindborg who coined the term PIOC in 1971 (1-2).

According to World Health Organisation (WHO) PIOC is defined as "A Squamous cell carcinoma arising with in the jaw, having no initial connection with the oral mucosa and presumably developing from residues of the odontogenic epithelium" (2). WHO classified the lesion as odontogenic carcinoma. There are several classifications but Waldron and Mustoe's (3) classification is widely accepted and frequently cited according to which PIOC may have different origins.

Type 1: PIOC ex odontogenic cyst

Type 2a: Malignant ameloblastoma

Type 2b: Ameloblastic carcinoma arising denovo, ex ameloblastoma or ex odontogenic cyst

Type 3: PIOC arising denovo

(a) Keratinizing type

(b) Non keratinizing type

Type 4: Intraosseous mucoepidermoid carcinoma

According to this classification, PIOC may have different origins. Total absence of cystic component or other odontogenic tumor cells such as ameloblastoma is mandatory to diagnose PIOC type 3 (de novo). But PIOC type 1 can be identified by the presence of odontogenic cyst. Similarly, PIOC type 2 can be distinguished by the presence of malignant ameloblastoma or ameloblastic carcinoma arising de novo. Discrimination between type-3a and $3 \mathrm{~b}$ PIOCs is based on the former lesion possessing keratin pearls and/or individual keratoses, whereas these features are absent in the latter. PIOC arising de novo must be considered if no cystic component of other odontogenic tumor cells is demonstrated. Although several cases of malignant transformation of odontogenic cysts have been reported in the literature while PIOC occurring denovo is rare (1-2).

To define a lesion in the jaws as PIOC, 3 specific criteria may be present (4-6):

(1) Histological evidence of squamous cell carcinoma,

(2) Absence of ulcer formation on the overlying mucosa, and

(3) Absence of a distant primary tumor at the time of diagnosis and at least 6 months during the follow-up period.

To eliminate the possibility of distant primary tumor concurrence, chest radiographs, bone scintigram, and endoscopy of the gastrointestinal system and upper respiratory tract should be performed during the diagnostic phase and follow-up period.

Etiology of PIOC is not clear probably it arises from the remnants of odontogenic tissues, either the epithelial rests of Malassez or the remnants of dental lamina.
These epithelial remnants proliferate and transform into odontogenic carcinoma, a process that is potentially triggered by an inflammatory process. It does not have its origin from the epithelial lining of a pre-existing odontogenic cyst or the epithelial component of an odontogenic tumor (2-3).

Tumor is locally aggressive and metastasis to lymph nodes. Prognosis is quite poor, with 5-year survival rates ranging from $30 \%$ to $40 \%$. Prognosis further worsens with delayed diagnosis and treatment. To et al. (7) has reported delays in correct diagnosis, ranging from a few weeks to as long as 18 months.

A case report with all the typical features is being presented here to improve the knowledge of all the readers in order to aid in early diagnosis and institution of suitable treatment to improve the prognosis.

\section{Case Report}

A 50 years old male patient reported to the Department of Oral Medicine and Radiology with a slightly painful swelling of right lower jaw of six months duration. Swelling appeared suddenly and it was not associated with any kind of pain. But presently, patient experiences sharp, intermittent pain. There is no increase in size of the swelling since six months.

He denied other medical problems or weight loss and stated that he was otherwise in a good health. History of smoking bidi was present since 30 years along with alcohol consumption socially. The rest of the family members were apparently normal.

On extraoral examination an oval shaped swelling was observed on facial aspect of right mandible about $2 \times 3 \mathrm{~cm}$ in size. Overlying skin was normal with no detectable lymphadenopathy of head and neck.

Intraoral examination revealed oval shaped, non tender, hard swelling about $1 \times 1 \mathrm{~cm}$ in size extendind from distal surface of 4.4 to mesial surface of 4.6 (Fig. 1). Loss of attachment of buccal mucosa was seen on buccal side of 4.6 along with grade one mobility. Overlying alveolar mucosa was intact without any evidence of a mass or ulcer. There was no nerve parasthesia. There was a pocket in relation to $4.5,4.6$. Area was bleeding easily on probing.

Panoramic radiograph demonstrated an ill defined radiolucent lesion extending from distal of 4.4 to mesial of 4.6 along with interdental bone loss around 4.6.

Under impression of periodontal abscess patient was sent to department of Oral Surgery for extraction of 4.5 and 4.6. The sockets were sutured after extraction. Patient again reported after 15 days with aggravated pain and no reduction in swelling. Panoramic radiograph was repeated which revealed a larger ill defined radiolucent lesion in the area of 4.5 and 4.6 with pathological fracture of lower border of mandible (Fig. 2). Then intraosseous carcinoma was suspected and incisional biopsy 


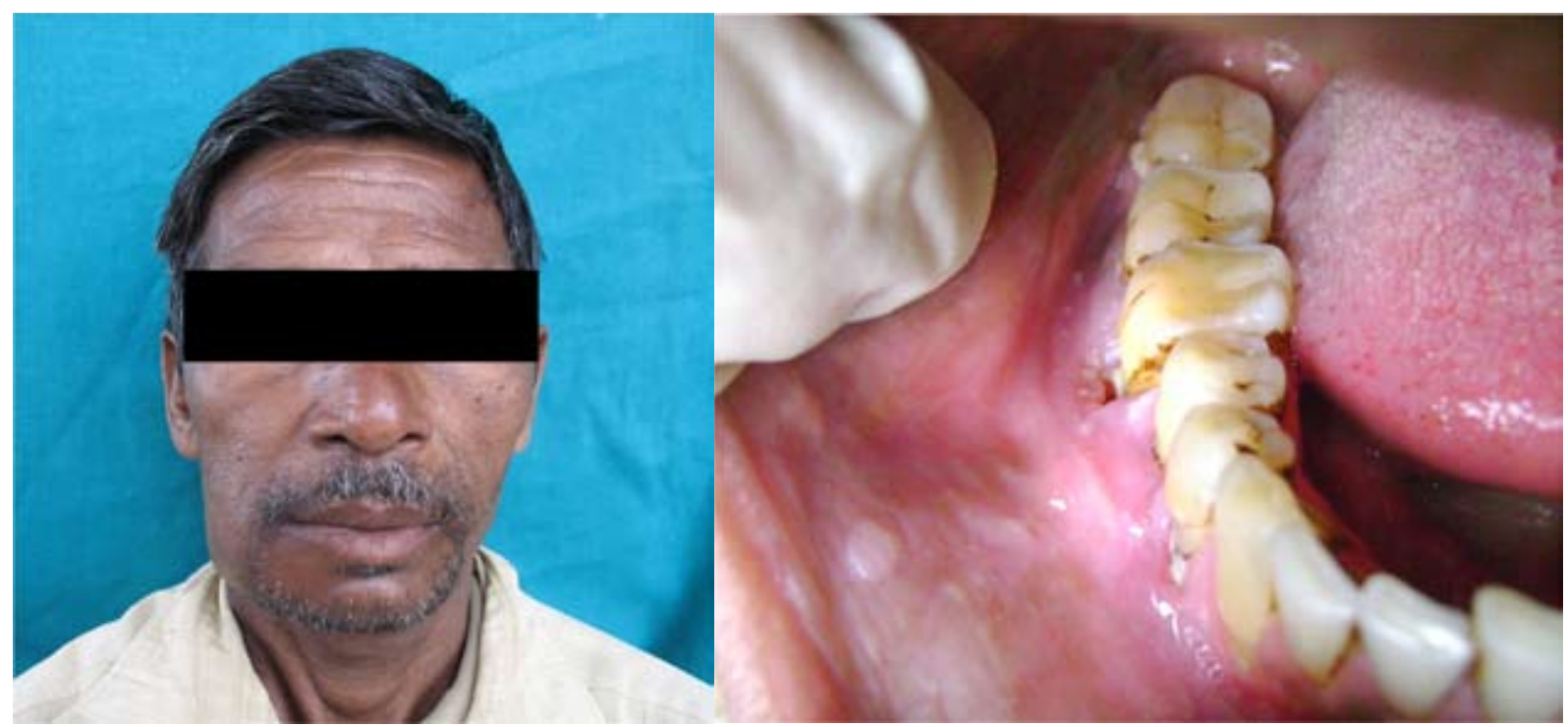

Fig. 1A. Showing facial asymmetry with a swelling on right side of the mandible.

Fig. 1B. Showing swelling in right mandibular sulcus along with gingival recession in relation to 4.6.

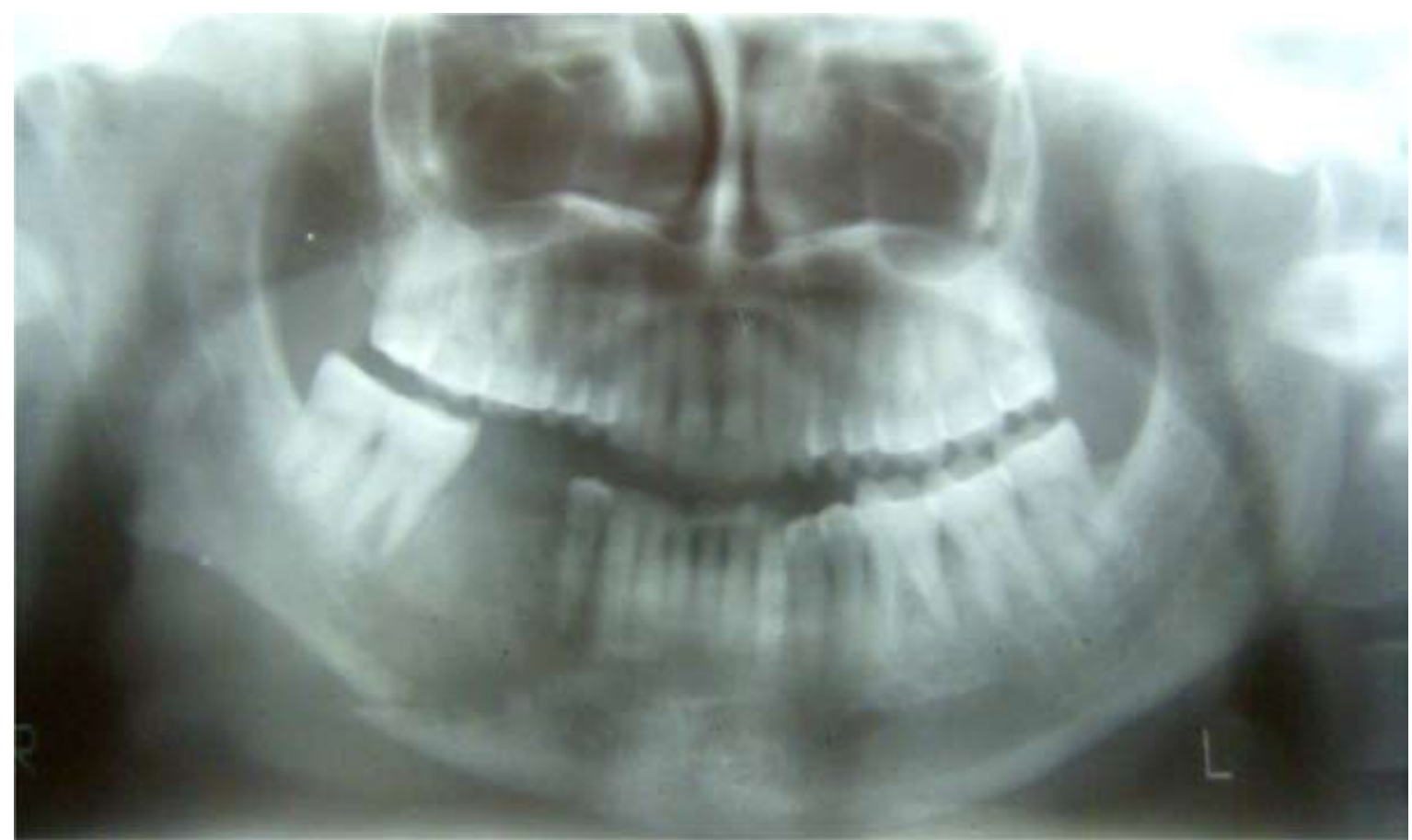

Fig. 2. Post treatment panoramic radiograph revealing larger ill defined radiolucent lesion in the area of 4.5 and 4.6 with pathological fracture of lower border of mandible.

was taken. Careful general physical examination was done to rule out distant metastasis.

Histopathological findings

Histopathological examination with hematoxylin and eosin (H\&E) staining showed well differentiated squamous cell carcinoma. Keratin pearl formation and individual cell keratinization is seen in the islands and nests of cells resembling squamous cells along with dysplastic features (Fig. 3).

Final diagnosis of PIOC was made. Right hemimandibulectomy with neck dissection was advised.

\section{Discussion}

The total number of reported cases of PIOC is difficult to determine owing to insufficient data to conclusively support the diagnosis of some published cases. Approximately more than 150 cases of PIOC have been documented till now; consisting of more than 90 cases of PIOC type 1 and rest are PIOC type 3 (1). Present case falls in the category of type 3 as it meets all the criteria explained earlier.

PIOC affects patients ranging from 4-90 years of age with mean age of 57 years. It is more frequently loca- 


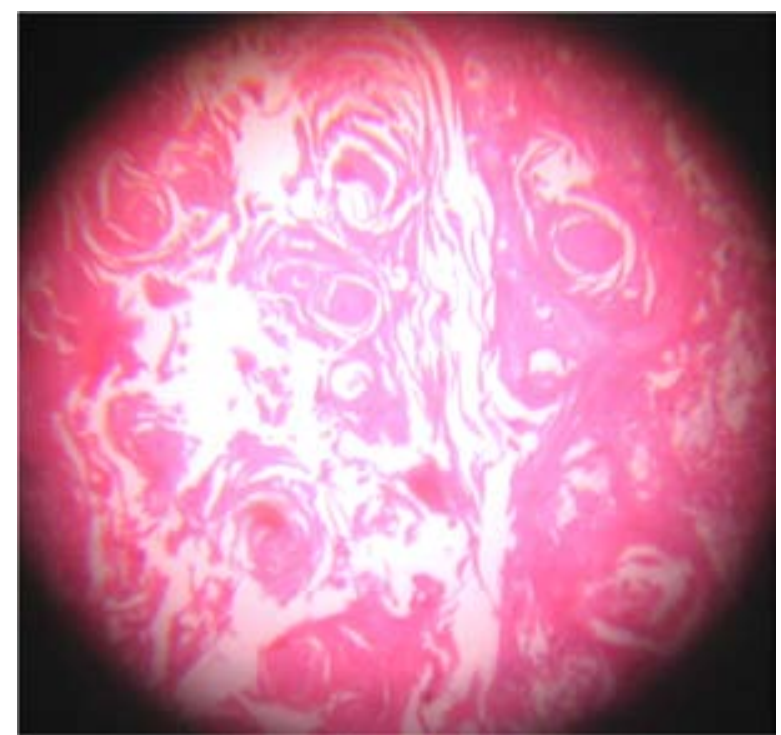

Fig. 3. Histopathological examination $H \&$ E staining showed well differentiated squamous cell carcinoma.

ted in mandible with a striking predilection for posterior regions. In maxilla lesions are mainly located in the anterior region and cross the midline. It affects men more than women with different ratios given in several studies (1-4).

Most common symptoms of patients with PIOC are pain and swelling to complete absence of subjective symptoms in early phases found on routine dental radiographs. There is progressive swelling of jaws and loosening of teeth. Accelerated growth with swelling, trismus, sensory disturbances such as parasthesia and numbness can also occur due to compression of inferior alveolar nerve in advanced cases (4). Spread to regional lymph nodes has been recorded in several cases. Microscopically, most of PIOC show the same histological features as squamous cell carcinoma.

Many studies show that $61 \%$ of PIOC presented a unilocular radiolucency resembling cyst varying in size shape and margins. In most of cases margins are irregular and poorly defined. These did not cause root resorption or displacement and tend to grow around obstructions as teeth rather than displace them, since invasion probably occurs along the path of least resistance.

Variability of radiographic features of PIOC and its resemblance to peri-apical lesions and radicular cysts, as well as other odontogenic cysts and tumors, emphasize that PIOC should be considered in the differential diagnosis of radiolucent lesions of jaws.

In case of periapical lesions caries or trauma will be present while radicular cyst will exhibit sclerotic margin. Malignant tumors of odontogenic epithelium, including ameloblastic carcinoma, intraosseous mucoepidermoid carcinoma, clear cell odontogenic carcinoma, odontogenic ghost cell carcinoma, and malignant variant of calcifying epithelial odontogenic tumour (CEOT) should be considered in differential diagnosis and squamous cell carcinoma of mucosal origin, acanthomatous ameloblastoma, squamous odontogenic tumor, and CEOT also should be ruled out.

Ameloblastic carcinoma demonstrates malignant features along with prominent peripheral palisading and reverse nuclear polarization which are absent in PIOC. The absence of a mucous component in PIOC, verified by a negative mucicarmine staining, serves to distinguish it from intraosseous mucoepidermoid carcinoma. A biphasic pattern characterized by clear cells and eosinophilic polygonal cells is the histopathologic hallmark of clear cell odontogenic carcinoma. In contrast, PIOC exhibits sheets or islands of malignant epithelial cells, with minimal or absent clear cell component. Odontogenic ghost cell carcinoma displays in addition to a malignant epithelial component the classic features of calcifying odontogenic cyst or tumor, including the presence of ghost cells and calcified material, which are only minimally present or completely absent in PIOC. Finally, most benign odontogenic tumors, in addition to features specific to each one of them, share a lack of invasive growth, with the exception of ameloblastoma and, to a lesser extent, CEOT, which may be locally invasive. Furthermore, cellular and nuclear atypia should be absent in a benign tumor except for the atypia seen in benign CEOT; the latter demonstrates additional diagnostic features, such as amyloid deposition and Liesegang ring calcifications. These findings also allow discrimination between PIOC and the very rare malignant variant of CEOT, which, in contrast to benign CEOT, exhibits a highly invasive growth pattern with vascular invasion or perineural spread (1).

Surgery is the treatment of choice and in most cases consisted of enbloc excision or radical resection of involved bone. Distraction osteogenesis of mandibular segmental defect may be a valid alternative in those patients who are not candidates for more aggressive surgical procedures. Radiotherapy and chemotherapy should be considered only in lesions that cannot be surgically controlled $(2-4)$.

Our case highlights that radiographic examination is one of the most effective methods for detecting early lesions of PIOCs. So if a patient is reporting clinically with asymptomatic swelling of jaw of long duration and radiographically an ill-defined osteolytic lesion is seen, PIOC should be ruled out before moving forward as it can prolong the life of a patient which is the main commitment of the dentistry to each of its individual. Being an oral diagnostician, the sole responsibility we can perform in case of PIOC is the early diagnosis. Hence, accurate knowledge of this rare entity is must to prevent delayed diagnosis.

\section{References}

1. González-García R, Sastre-Pérez J, Nam-Cha SH, Muñoz-Guerra 
MF, Rodríguez-Campo FJ, Naval-Gías L. Primary intraosseous carcinomas of the jaws arising within an odontogenic cyst, ameloblastoma, and de novo: report of new cases with reconstruction considerations. Oral Surg Oral Med Oral Pathol Oral Radiol Endod. 2007;103:e2933.

2. Thomas G, Pandey M, Mathew A, Abraham EK, Francis A, Somanathan T, et al. Primary intraosseous carcinoma of the jaw: pooled analysis of world literature and report of two new cases. Int J Oral Maxillofac Surg. 2001;30:349-55.

3. Waldron CA, Mustoe TA. Primary intraosseous carcinoma of the mandible with probable origin in an odontogenic cyst. Oral Surg Oral Med Oral Pathol. 1989;67:716-24.

4. Zwetyenga N, Pinsolle J, Rivel J, Majoufre-Lefebvre C, Faucher A, Pinsolle V. Primary intraosseous carcinoma of the jaws. Arch Otolaryngol Head Neck Surg. 2001;127:794-7.

5. Suei Y, Tanimoto K, Taguchi A, Wada T. Primary intraosseous carcinoma: review of the literature and diagnostic criteria. J Oral Maxillofac Surg. 1994;52:580-3.

6. Lin YJ, Chen $\mathrm{CH}$, Wang WC, Chen YK, Lin LM. Primary intraosseous carcinoma of the mandible. Dentomaxillofac Radiol. 2005;34:112-6.

7. To EH, Brown JS, Avery BS, Ward-Booth RP. Primary intraosseous carcinoma of the jaws. Three new cases and a review of the literature. Br J Oral Maxillofac Surg. 1991;29:19-25. 